\title{
Aeroacoustic Characteristics of Model Jet Test Facility Flow Conditioners
}

\author{
Kevin W. Kinzie ${ }^{*}$, Brenda S. Henderson ${ }^{* *}$, and Harry H. Haskin ${ }^{* * *}$ \\ NASA Langley Research Center, Hampton, VA 23681
}

\begin{abstract}
An experimental investigation of flow conditioning devices used to suppress internal rig noise in high speed, high temperature experimental jet facilities is discussed. The aerodynamic and acoustic characteristics of a number of devices including pressure loss and extraneous noise generation are measured. Both aerodynamic and acoustic characteristics are strongly dependent on the porosity of the flow conditioner and the closure ratio of the duct system. For unchoked flow conditioners, the pressure loss follows conventional incompressible flow models. However, for choked flow conditioners, a compressible flow model where the duct and flow conditioner system is modeled as a convergent-divergent nozzle can be used to estimate pressure loss. Choked flow conditioners generate significantly more noise than unchoked conditioners. In addition, flow conditioners with small hole diameters or sintered metal felt material generate less "self-noise" noise compared to flow conditioners with larger holes.
\end{abstract}

$\begin{array}{ll}A R & =\text { duct closure ratio } \\ C B & =\text { counterbored flow conditioner } \\ d_{1} & =\text { flow conditioner minimum hole diameter } \\ d_{2} & =\text { flow conditioner counterbore hole diameter } \\ F C P R & =\text { flow conditioner pressure ratio } \\ O P R & =\text { operating pressure ratio } \\ P O A & =\text { percent open area; porosity } \\ l_{1} & =\text { flow conditioner counterbore depth } \\ L & =\text { flow conditioner thickness } \\ \text { mic } & =\text { flow conditioner with microholes } \\ K & =\text { pressure loss coefficient } \\ S t & =\text { flow conditioner with straight through holes; no counterbore } \\ S t_{D} & =\text { Strouhal number based on exit orifice diameter and velocity } \\ S t_{f c} & =\text { Strouhal number based on flow condition hole diameter and velocity } \\ U & =\text { flow velocity } \\ \theta & =\text { directivity angle } \\ \rho & =\text { density }\end{array}$

\section{Introduction}

Obtaining high quality acoustic data from facilities designed to measure jet noise requires great care in the delivery of flow to the test chamber. Noise generated in piping can severely contaminate far-field acoustic measurements. Several recent works have examined potential sources of internal rig noise, their impact on acoustic measurements, and design guidelines to quiet these noise sources ${ }^{1,2}$. One of the most effective ways to suppress internal noise is to provide a large pressure drop close to the model system exit so that upstream pipe flow velocities and resulting flow noise are both reduced. In addition, the sudden pressure drop creates an impedance change that alters the propagation of sound waves through the flow duct. Pressure drops are also often required when the test

\footnotetext{
${ }^{*}$ Research Scientist, Aeroacoustics Branch, Hampton, VA, MS 166, AIAA Senior Member

${ }^{* *}$ Research Scientist, Aeraocoustics Branch, Hampton, VA, MS 166, AIAA Member

${ }^{* * *}$ Engineer, Aeraocoustics Branch, Hampton, VA, MS 166, AIAA Member
}

1

American Institute of Aeronautics and Astronautics 
stand is supplied with air from a high-pressure supply line or when flow conditioning is required to provide uniform velocity and turbulence conditions to the model system.

Choke plates, screens, porous materials, and high-density mesh material are commonly used to provide pressure drop and flow conditioning. However, many devices that provide sufficient pressure drop also create a great amount of "self-noise". Self-noise is defined as noise related directly to the presence of the flow conditioner and includes sources such as high-speed jet flow exiting individual holes in the flow conditioner or turbulent mixing in the flow duct as the flow adjusts downstream of the flow conditioner. Choke plates of some kind, either in the form of a single-hole orifice plate or a plate with multiple small holes, are well suited to provide pressure loss because they are relatively easy to manufacture and can be designed to withstand high pressure and temperature loading often imposed by test facilities. However, they are also inherently very noisy. While choke plates can reduce the upstream approach velocity, flow through the choke plate often reaches sonic conditions and can generate shock-associated noise. If the characteristic length scales of the turbulence generated by the choke plate holes are sufficiently small compared to the length scale of the turbulence generated by the jet model, the extraneous noise from the choke plate may not be a problem in far-field acoustic measurements.

Porous material and high-density mesh screens can also provide pressure drop without as much extraneous noise as choke plates with larger holes because they generate turbulent length scales that are very small and dissipate quickly or generate noise of such high characteristic frequency as to not contaminate the frequencies of interest. Consequently, they typically have better acoustic qualities compared to choke plates. However, it is more difficult to design a flow conditioner of this type that can withstand the required high temperature and pressure loading. In facilities where liquid fuels are used for combustion, unburned fuel can also wick into the mesh materials and ignite in an undesirable fashion. In addition, when seeding the flow for optical measurement techniques such as Particle Image Velocimetry (PIV), the porous material will quickly clog with seed material.

With all of the previously mentioned characteristics in mind, a desirable flow conditioning device for a high speed, high temperature jet noise rig is one that provides the right amount of pressure drop in the model system without introducing extraneous noise sources to the final acoustic measurements. It should be strong enough to withstand pressure and temperature loading without deforming, but porous enough to allow optical seeding material on the order of 1 micron to pass without clogging. While the flow passages need to be large enough not to clog with seed particles, they cannot be too large so as to generate noise in an undesirable frequency range. Clearly, it is difficult to identify a single flow conditioning device that meets all of these constraints. The problem is compounded by the fact that there is very little information in the open literature that provides pressure drop characteristics in the flow regime usually encountered in a jet noise experimental facility and even less information on acoustic characteristics of flow conditioning devices. Reference 3 is a good source for making pressure loss calculations in compressible flow, but does not cover the range of thicker plates measured here and provides no information regarding acoustic characteristics.

This paper reports an investigation into flow conditioning devices with the potential for use in high speed, high temperature experimental jet facilities. Several choke plate designs with various hole sizes and geometry are evaluated for pressure drop and noise generation characteristics. In addition, a porous metal felt mesh material is also investigated.

\section{Experimental Approach}

Experiments were conducted in the Small Anechoic Jet Facility (SAJF) in NASA Langley's Jet Noise Laboratory (JNL). A $50.8 \mathrm{~mm}$ diameter flow duct test apparatus was constructed that allows various test samples to be installed and evaluated over a range of operating pressures. The flow duct exhausts through a converging orifice plate into an anechoic chamber where acoustic measurements are made. Simultaneous pressure drop measurements are also made across the flow conditioner.

A schematic and photograph of the test apparatus are shown in Fig. 1. A $152.4 \mathrm{~mm}$ diameter settling chamber converges to a straight $50.8 \mathrm{~mm}$ duct where the test sample/flow conditioner is installed. Downstream of the test sample, the $50.8 \mathrm{~mm}$ duct continues for $152.4 \mathrm{~mm}$ until it reaches a converging orifice plate where several different diameter orifices were installed to generate different pressure and flow conditions inside the duct and through the flow conditioner. All piping was hard wall with no acoustic treatment of any kind. Total and static pressure measurements were made upstream and downstream of the flow conditioner.

Acoustic measurements were made by an eight-microphone sideline array located approximately $2.44 \mathrm{~m}$ from the nozzle centerline axis. Microphones were $1 / 4$ " diameter, operated with the grid caps removed, and calibrated with a pistonphone and electrostatic calibrator. The data are scaled to a $3.66 \mathrm{~m}$ arc and corrected to the same reference day atmospheric conditions for comparison. 
An important parameter in establishing pressure and velocity conditions around the flow conditioner is the closure ratio of the duct system. The closure ratio, (AR), is defined as the area ratio of the duct exit orifice and the flow duct cross-section at the location of the flow conditioner. Higher values of AR result in higher flow velocity and lower static pressure inside the duct and upstream from the exit. Each flow conditioner was tested with three values of closure ratio $(\mathrm{AR}=0.3,0.43$, and 0.56 ). These specific ratios were chosen because they are representative of nozzle systems tested in the larger NASA Low Speed Aeroacoustic Wind Tunnel jet noise facility. The actual diameter of these orifice nozzles were $27.8 \mathrm{~mm}, 33.3 \mathrm{~mm}$, and $38.1 \mathrm{~mm}$.

A schematic of the flow conditioner cross-sections is shown in Fig. 2 and Table 1 shows salient dimensions of each test sample. Photographs of select flow conditioners are shown in Fig. 3. As will be seen in the data, the primary factor influencing the flow conditioner characteristics is the total porosity of the conditioner. The porosity is characterized by the percent open area (POA) of the sample and is defined as the total open area of the flow conditioner divided by the flow duct area at the location of the flow conditioner. Two hole sizes were evaluated; $2.39 \mathrm{~mm}$ diameter and $0.889 \mathrm{~mm}$ diameter. The larger holes were slightly countersunk on the upstream side to reduce entrance losses, but the smaller holes had sharp entrances and exits. The effect of counterboring the downstream side to improve mixing characteristics of the individual jets emanating from each of the holes was evaluated for the larger size holes (conditioners 1, 3, and 6). The effect of hole geometry for a given POA and thickness was explored at 16.5 POA (conditioners 3, 4, and 5). The three different hole geometries and counterbore were measured with $12.7 \mathrm{~mm}$ thick plates. The four highest porosity plates had a larger thickness of $25.4 \mathrm{~mm}$. In addition to the choke plate style flow conditioner, a sintered metal felt material was also tested.

The experiments were conducted by setting a range of operating pressure ratios (OPR) and measuring total and static pressure conditions upstream and downstream of the flow conditioner and the noise radiated into the anechoic chamber. The OPR is defined as the ratio of the total pressure of the flow exiting the duct $\left(\mathrm{Pt}_{2}\right.$ in Fig. 1$)$ divided by the ambient static pressure. The total temperature of the flow was held constant at $311^{\circ} \mathrm{K}$ for every test point. The pressure drop across the flow conditioner is defined as the difference between the total pressure in the plenum upstream of the flow conditioner $(\mathrm{Pt})$ and the total pressure measured just upstream of the convergent duct orifice $\left(\mathrm{Pt}_{2}\right)$.

\section{Pressure Measurement Results}

The pressure losses across all of the flow conditioning devices are shown as a function of OPR in Fig. 4. These data are for $A R=0.43$. Data for the other closure ratios have similar trends. The amount of pressure loss across the flow conditioners increases monotonically as the porosity decreases. For the 9.5 POA device, OPRs above 2 could not be set because of the significant upstream pressure required. In addition, the effect of the counterbore as measured on the 16.5 POA device had only a small impact on the pressure loss. However, the smaller hole size had a more measurable effect on this porosity flow conditioner. As will be shown later, it is likely that frictional loss through the much smaller holes is higher and results in an effective porosity less than the geometric porosity. It is also observed that as the exit orifice approaches the choke point of OPR $=1.89$ for all cases, the pressure drop becomes a linear function of OPR. In fact, a linear approximation of pressure loss is a reasonable estimate down to $\mathrm{OPR}=1.6$ and is within about $10 \%$ of the linear value down to $\mathrm{OPR}=1.4$.

In order to determine if the flow conditioners are choking, the flow conditioner pressure ratio (FCPR) for all of the devices are plotted in Fig. 5. The FCPR is defined as the ratio of total pressure upstream of the flow conditioner (Pt) divided by the static pressure in the duct downstream of the flow conditioner $\left(\mathrm{Ps}_{2}\right)$. A FCPR above 1.89 implies choked flow through the flow conditioner. It is interesting to note that the FCPR quickly reaches a constant value and then does not change as OPR increases. Only data for $\mathrm{AR}=0.43$ are shown, but the other closure ratios have similar trends. For the smaller closure ratio of $\mathrm{AR}=0.3$, the FCPR is generally lower than that shown in Fig. 5 and for $\mathrm{AR}=0.56$ the FCPR is higher. The sintered mesh material shows a slight decrease in FCPR with increasing OPR. However, it is believed that as the upstream pressure forces increased on the mesh, the mesh deformed slightly and allowed more leakage around the edges thereby effectively changing the flow conditioner POA as OPR increased. For this closure ratio, all flow conditioner devices with a POA of 25 and lower are choked at all conditions with the exception of the 25 POA device at the lowest measured OPR of 1.2. Devices with porosity higher than $25 \%$ are unchoked at all conditions.

Figure 6 shows a plot of the maximum FCPR attained as a function of flow conditioner POA for all three values of closure ratio. As mentioned earlier, smaller closure ratios result in lower values of FCPR. For POA values between 25 and 43.7, whether or not the flow conditioner chokes is a function of the duct closure ratio. Figure 7 shows that if the $\mathrm{FCPR}_{\max }$ values shown in Fig. 6 are normalized by the closure ratio they nearly collapse to a single 
curve except for the low closure ratio and high porosity configurations. In general, the data for choked flow conditioners collapse better than those that do not. The physical mechanism that results in this behavior can be described using compressible flow relations and will be discussed shortly.

Before further exploring the pressure loss phenomenon for the choked flow conditioners, we will examine the unchoked cases. Figure 8 shows the pressure drop curve for the 40.7 POA flow conditioner, which was barely choked for $\mathrm{AR}=0.56$, but unchoked for the lower closer ratios. As expected, the pressure loss increases for higher closure ratios. Since this porosity does not follow the same FCPR trend as the higher compressibility cases, let us consider a more conventional pressure loss model for incompressible flow. There are many derivations in the literature to model the pressure drop across an orifice ${ }^{4,5}$, but most generally describe a flow coefficient, $\mathrm{K}$, of the form:

$$
\mathrm{K}=\left(\frac{\mathrm{Pt}_{1}-\mathrm{Pt}_{2}}{\frac{1}{2} \rho_{1} \mathrm{U}_{1}^{2}}\right)
$$

If the pressure coefficient in equation (1) is normalized by the closure ratio, AR, and the pressure drop data of Fig. 8 are replotted, as shown in Fig. 9, it is observed that there is a general collapse of the data indicating that the incompressible model works reasonably well for this flow conditioner. If the data from other flow conditioner devices at all closure ratios are plotted in a similar fashion, as shown in Fig. 10., it is seen that the ability of equation (1) to normalize the pressure loss becomes less effective as the porosity of the flow conditioner becomes lower, or as the compressibility effects become more significant as indicated by the FCPR. For the highest porosity devices of 47.6 and 51.7 POA, the normalized pressure loss coefficients are nearly identical. As the porosity decreases, and the pressure ratio across the flow conditioner increases, the spread in the normalized pressure loss data also increases. For 16.5 POA, there is a very poor correlation of K/AR for different closure ratios. The conclusion from these observations is that, for the incompressible flow regime, the pressure loss through these types of flow conditioners can be described reasonably well described by conventional models of pressure loss. However, as the flow conditioner porosity is reduced and the flow accelerates through the device, compressibility effects become more important and another pressure loss model is required.

Returning to Fig. 7, an explanation of why the unchoked values of FCPR do not follow the same trend as the choked values has been provided. However, the good collapse of the remaining data implies that there is also a physical model that describes the behavior for the choked cases. Since the flow conditioner is a choke point and the flow is sonic at that location, the flow will expand as it exits the flow conditioner. Since the flow must adjust to the cross-sectional area of the duct downstream of the flow conditioner, the flow duct upstream and downstream of the flow conditioner might be modeled as a convergent-divergent nozzle system with the flow conditioner being the throat. In this model, it is assumed that the CD nozzle has a shock standing in the divergent portion of the nozzle. For different closure ratios, the pressure at the exit of the divergent section would change and thereby cause differences in the total pressure loss through the system as the shock location changed for each closure ratio.

In order to evaluate this hypothesis, the geometry of the flow duct system will be modeled as shown in Fig. 11. The total pressure at points 1 and 4 are known and the system will be evaluated for a choked orifice condition at point 5, which has a known cross-sectional area. Since the stagnation pressure ratio across the hypothetical shock wave is known from the measurements in the flow duct, the strength of the shock is known and the hypothetical shock location can be found using isentropic and normal shock relations. It is then a straightforward process to use the actual choked flow area at the duct exit to calculate the choked flow area of the throat at location 2 . If the model is valid, the throat area should be approximately equal to the area of the flow conditioner. This process of calculating the flow conditioner area based on the measured pressure conditions was followed for a number of the flow conditioner and orifice configurations and the results are summarized in Fig. 12. The flow conditioner porosity calculated using this method is plotted against the actual geometric flow conditioner porosity. Note that due to frictional losses, entrance losses, and manufacturing issues, the actual effective POA is expected to be less than the nominal geometric POA. However, it is not expected that the actual effective POA will change significantly over the range of closure ratios although it could be slightly dependent on Reynolds number. Therefore, an inconsistently calculated porosity over the range of closure ratios implies a breakdown of the inviscid compressible flow model. For flow conditioners where choking is expected, the model works extremely well. For flow conditioners that are unchoked, the model does not give a consistent calculation of POA. The conclusion is that this method can be used very effectively to model the pressure loss across a given flow conditioner provided the flow conditioner is choked and the effective porosity is known or can be estimated.

One more observation from Fig. 12 is that the difference between the effective porosity of the flow conditioner compared to the geometric porosity is strongly dependent on the details of the hole geometry. Table 2 
shows the geometric POA of several flow samples compared to the actual effective POA calculated with the choked flow model with the largest closure ratio of $\mathrm{AR}=0.56$. There is a difference for the two different hole sizes of the 16.5 POA conditioner with the lower porosity of the smaller holes attributed to larger friction losses. The 40.7 POA flow conditioner is included in this table because the pressure measurements indicate that it is choked for $\mathrm{AR}=0.56$ so the estimate of effective POA should be valid. There is a much larger difference between the geometric and effective porosity for this conditioner presumably because of the extra thickness of this plate and therefore, increased effects of friction. Additionally, while it is difficult to physically measure the geometric porosity for the sintered mesh material, this method can be used to determine an effective porosity of the material that could then be used to estimate the pressure loss in another application.

\section{Acoustic Results}

In addition to characterizing the aerodynamic properties of these flow conditioners, it is important to characterize their acoustic qualities and determine the likelihood that they would contribute extraneous noise if used in a jet noise test application. While acoustic measurements were made at several sideline locations, only measurements at the sideline angle of $\theta=90^{\circ}$ will be shown here. Extraneous noise tends to be more of a contributor to the overall sound levels in this direction compared to more aft angles where the peak jet noise levels can mask other noise sources. In order to identify extraneous noise added to the system, it is important to understand the inherent noise levels of the system in the absence of the flow conditioners. Figure 13 shows the baseline spectra for four different nozzle closure ratios at $\mathrm{OPR}=1.8$ with no flow conditioners installed. The data are scaled to frequency and amplitude levels of the AR $=0.56$ orifice using the method described in reference 6 . There is excellent agreement of the data for most of the spectral range. At high frequency, there are slightly higher noise levels for the larger nozzles, but in general these measurements give confidence that valid comparisons can be made between the flow conditioners and different closure ratios and these spectra are used as baseline references.

Far-field acoustic measurements for all of the flow conditioners and the baseline orifice at OPR $=1.8$ are shown for closure ratios of $\mathrm{AR}=0.3,0.43$, and 0.56 in Figs. 14, 15, and 16, respectively. The data are shown on an absolute frequency scale with no amplitude adjustment. The data for OPR $=1.8$ are representative of those at other operating conditions. However, there are some differences that should be explored in future work. For example, the source of the strong tones observed in some cases was originally thought to be a result of a resonance related to the counterbore built into some of the flow conditioners. However, more detailed examination of the data at other operating conditions indicates that it is a more complex resonance of the system possibly associated with the nozzle orifice plate or a coupling between the flow conditioner and orifice plate. It is believed that general conclusions about the overall suitability for using these flow conditioners in a jet noise facility can still be assessed even without a full explaination of these tones.

From Figs. 14-16, it is clear that the flow conditioners have the potential to generate extremely high levels of excess noise that significantly contaminate the pure jet noise signature. In addition, the closure ratio plays a strong role in the internal noise characteristics of the system. In general, choking the flow conditioner results in a large amount of excess noise. A porosity of $25 \%$ or lower results in a choked condition for all closure ratios except for the $25 \mathrm{POA}$ flow conditioner at $\mathrm{AR}=0.3$. For the flow conditioners with $2.39 \mathrm{~mm}$ diameter holes, the closure ratio trend of the relative noise levels above the baseline is indeterminate because of the aforementioned resonance tones. However, for flow conditioners with the smaller holes and for the sintered mesh, the relative excess noise levels above the baseline clearly increases as closure ratio increases. This is an intuitive result because the internal velocity at every point through the system also increases as closure ratio increases and aeroacoustic noise sources generally scale with some power of the velocity. Note also that while the sintered mesh material has higher noise levels than the high porosity microhole choke plates, the excess noise level is much less than the other choke plates with similar porosities. If required for a specific application, the mesh material can provide high levels of pressure loss with relatively low excess noise.

In addition to providing pressure loss, the flow conditioners will cause an impedance change in the duct so that some of the noise propagating from upstream will not be transmitted through to the exit of the duct. A direct measurement of impedance or insertion loss is not available from these data, but observe that for the closure ratios of 0.3 and 0.43 , high frequency noise levels for porosities higher than $43.9 \%$ are slightly less than the baseline. This implies that these flow conditioners are supplying some amount of suppression for upstream generated rig noise that is not observed in the jet acoustic signature. However, for the highest closure ratio of 0.56 , noise levels of the high porosity conditioners has increased over the baseline presumably from the higher flow velocities generated at the exit of the flow conditioner. Direct measurement of the insertion loss provided by these types of flow conditioning devices are planned for the future. 
Recall that the 16.5 POA conditioner was tested with three different hole geometries to explore the effect on pressure loss and noise. Acoustic data for the three configurations are compared to the baseline for a closure ratio of 0.43 and OPR $=1.8$ in Fig. 17. The counterbored holes are the loudest and the microholes are the quietest. A very significant observation is that even though the microholes are 2.7 times smaller than the larger holes, the peak frequency of the excess noise for both cases is very similar. The implication is that the noise radiated from flow conditioners of this type does not scale on the diameter of the individual holes. It is likely that the array of tiny jets exhausting from the holes merge very quickly downstream so that the radiated noise cannot be characterized by the size of the individual holes. In order to examine this phenomenon closer, the acoustic spectra of Fig. 16 were frequency scaled using the fully expanded velocity conditions at the flow conditioner exit and by the diameter of the individual hole. Figure 18 shows the acoustic data scaled in this manner. For an individual jet, the peak Strouhal number based on jet diameter is typically $0.2-0.4$. The larger hole conditioners clearly show peak frequencies in this range. For the microhole choke plates for porosities above $25 \%$, the small amount of excess noise observed compared to the baseline in Fig. 16 also roughly falls into this range. However, for the 16.5 POA microhole choke plate, the peak frequency is much lower than the others. It is possible that the choked condition for this plate results in an acoustic field with length scales that are not well represented by the small holes. This would be consistent with the observed aerodynamic characteristic that this choke plate/duct system behaves more like a single convergent-divergent nozzle than as an array of small supersonic jets.

\section{Conclusion}

The following general design practices can be determined from these results.

1. For flow conditioning devices that are unchoked, traditional pressure loss models appear applicable.

2. For flow conditioning devices that are choked, the pressure loss can be modeled by treating the flow duct and flow conditioner as a convergent-divergent nozzle system and calculating the pressure loss associated with a normal shock wave standing in the divergent section.

3. Choking the flow conditioners creates large levels of extraneous noise and should be avoided unless there is a way to attenuate the shock-associated noise.

4. The thick microhole choke plates with high porosity and low pressure drop appear to provide beneficial acoustic impedance for small closure ratios, but have the potential to also generate excess noise as the closure ratio increases. The implication is that a series of unchoked high porosity flow conditioners would be the best implementation if large pressure loss is required with low excess noise generation.

5. The characteristic length scale associated with the excess noise appears to scale reasonably well with the flow conditioner hole size for the larger hole size and the unchoked microholes. However, for the choked microholes, the characteristic length scale is similar to that of the larger hole size.

6. The sintered mesh material provided large values of pressure drop with much lower noise levels compared to the choke plate with holes of similar porosity.

\section{Acknowledgements}

The authors thank the staff of the NASA Langley Jet Noise Laboratory for their assistance and hard work in fabricating the hardware and running the experiment. In addition, James Bridges and John Wolter of NASA Glenn Research Center provided helpful insights and loaned flow conditioning test samples for this work.

\section{References}

${ }^{1}$ Ahuja, K.K., "Designing Clean Jet Noise Facilities and Making Accurate Jet Noise Measurements," 2003, Int. J. of Aeroacoustics, Vol. 2, No. 3 \& 4, pp. 371-412.

2 Viswanathan, K,. "Jet Aeroacoustic Testing Issues: Issues and Implications," 2003, AIAA Journal, Vol. 41, No. 9, pp. 1674-1689.

3 Engineering Sciences Data Unit (ESDU) Report 82009, "Compressible flow of gases."

${ }^{4}$ White, F.M, Fluid Mechanics, 1979, McGraw-Hill, Inc.

5 Ward-Smith, A.J., Internal fluid flow: The fluid dynamics of flow in pipes and ducts, 1980, Clarendon Press.

${ }^{6}$ Soderman, P.T., and Allen, C.S., "Microphone measurements in and out of airstreams," Aeroacoustic Measurements, Springer 2002, pp. 1-61. 
Table I Flow conditioner characteristics.

\begin{tabular}{|c|l|c|c|c|c|c|}
\hline $\begin{array}{c}\text { Config. } \\
\text { Number }\end{array}$ & \multicolumn{1}{|c|}{ Description } & $\begin{array}{c}\text { Nominal } \\
\text { POA }\end{array}$ & $\begin{array}{c}\mathrm{d}_{1} \\
(\mathrm{~mm})\end{array}$ & $\begin{array}{c}\mathrm{d}_{2} \\
(\mathrm{~mm})\end{array}$ & $\begin{array}{c}\mathrm{l}_{1} \\
(\mathrm{~mm})\end{array}$ & $\begin{array}{c}\mathrm{L} \\
(\mathrm{mm})\end{array}$ \\
\hline 1 & counterbored holes & 9.5 & 2.39 & 4.78 & 7.62 & 12.7 \\
\hline 2 & sintered metal felt mesh & $\mathrm{n} / \mathrm{a}$ & $\mathrm{n} / \mathrm{a}$ & $\mathrm{n} / \mathrm{a}$ & $\mathrm{n} / \mathrm{a}$ & 0.381 \\
\hline 3 & counterbored holes & 16.5 & 2.39 & 4.78 & 7.62 & 12.7 \\
\hline 4 & straight large holes & 16.5 & 2.39 & $\mathrm{n} / \mathrm{a}$ & $\mathrm{n} / \mathrm{a}$ & 12.7 \\
\hline 5 & straight microholes & 16.5 & 0.889 & $\mathrm{n} / \mathrm{a}$ & $\mathrm{n} / \mathrm{a}$ & 12.7 \\
\hline 6 & counterbored holes & 25 & 2.39 & 4.78 & 7.62 & 12.7 \\
\hline 7 & straight microholes & 40.7 & 0.889 & $\mathrm{n} / \mathrm{a}$ & $\mathrm{n} / \mathrm{a}$ & 25.4 \\
\hline 8 & straight microholes & 43.9 & 0.889 & $\mathrm{n} / \mathrm{a}$ & $\mathrm{n} / \mathrm{a}$ & 25.4 \\
\hline 9 & straight microholes & 47.6 & 0.889 & $\mathrm{n} / \mathrm{a}$ & $\mathrm{n} / \mathrm{a}$ & 25.4 \\
\hline 10 & straight microholes & 51.7 & 0.889 & $\mathrm{n} / \mathrm{a}$ & $\mathrm{n} / \mathrm{a}$ & 25.4 \\
\hline
\end{tabular}

Table 2 Porosity calculated using compressible flow model.

\begin{tabular}{|c|c|c|}
\hline $\begin{array}{c}\text { Config. } \\
\text { Number }\end{array}$ & $\begin{array}{c}\text { Nominal } \\
\text { Geometric } \\
\text { POA }\end{array}$ & $\begin{array}{c}\text { Calculated } \\
\text { POA }\end{array}$ \\
\hline 1 & 9.5 & 7.7 \\
\hline 2 & $\mathrm{n} / \mathrm{a}$ & 10.6 \\
\hline 4 & 16.5 & 15.1 \\
\hline 5 & 16.5 & 13.1 \\
\hline 6 & 25 & 21.9 \\
\hline 7 & 40.7 & 28.6 \\
\hline
\end{tabular}



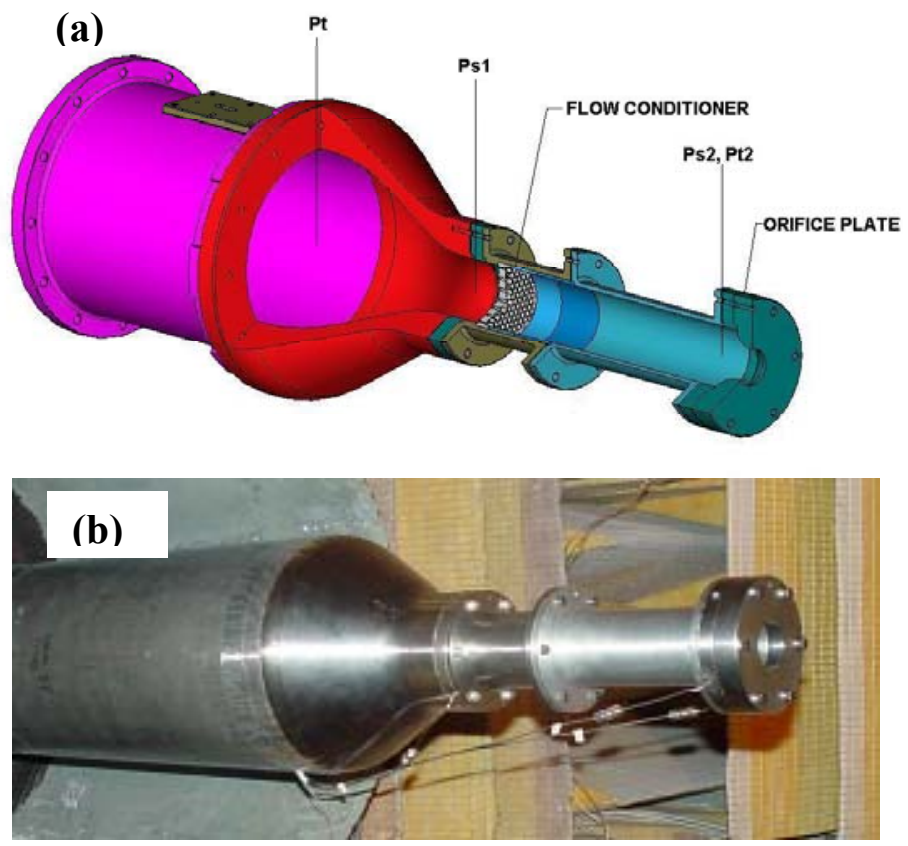

Figure 1. (a) Schematic of flow duct assembly and pressure measurement locations; (b) Photograph of flow duct installed in the SAJF.
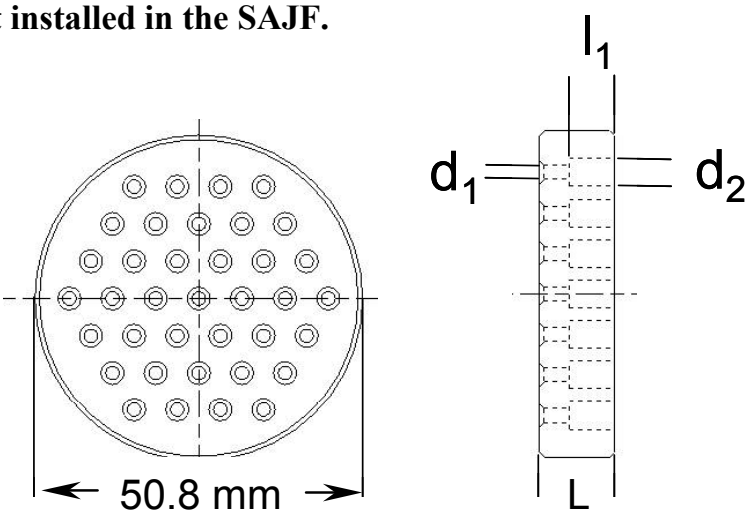

Figure 2. Schematic of flow conditioner devices. Dimensions are provided in Table $I$.

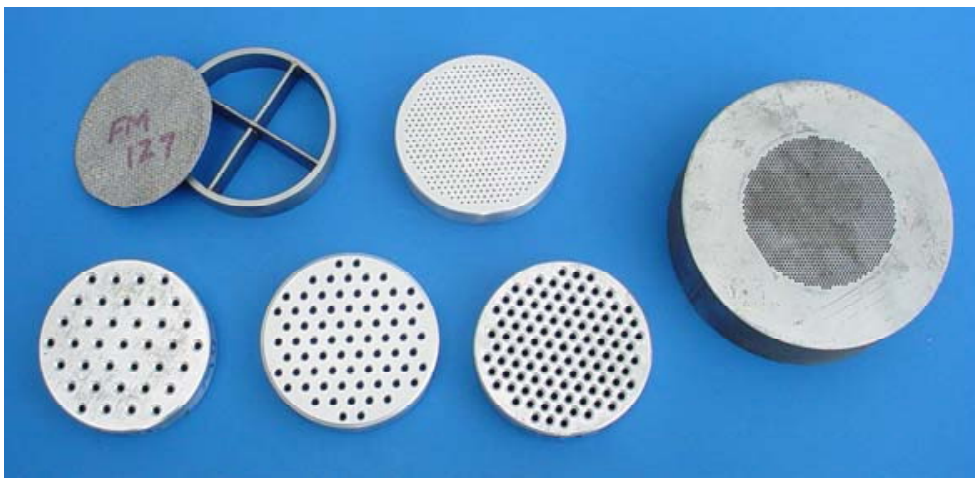

Figure 3. Photograph and configuration number (see Table 1) of select flow conditioners.

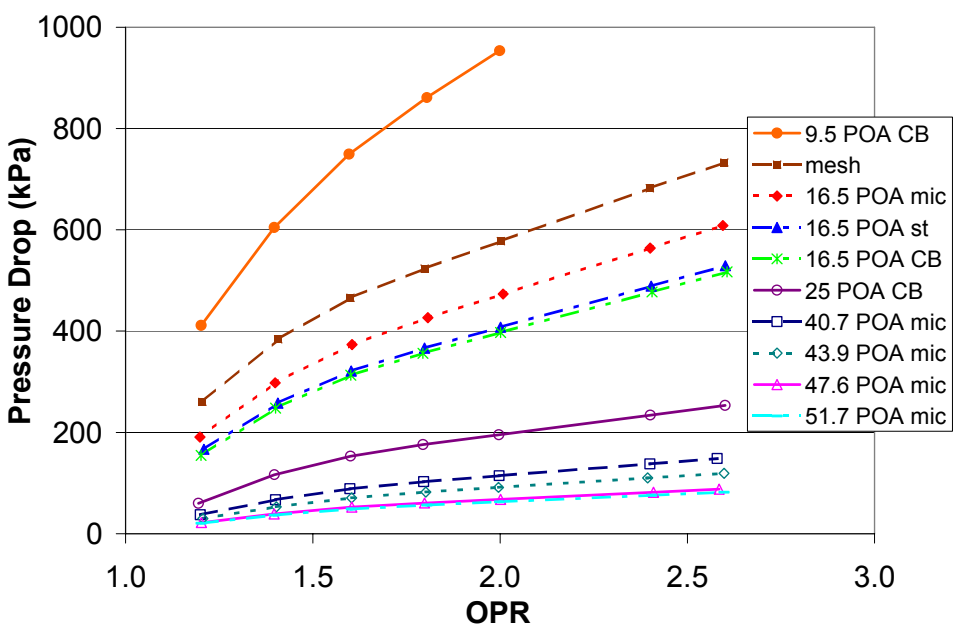

Figure 4. Stagnation pressure loss versus orifice pressure ratio for all flow conditioning devices at $\mathrm{AR}=\mathbf{0 . 4 3}$.

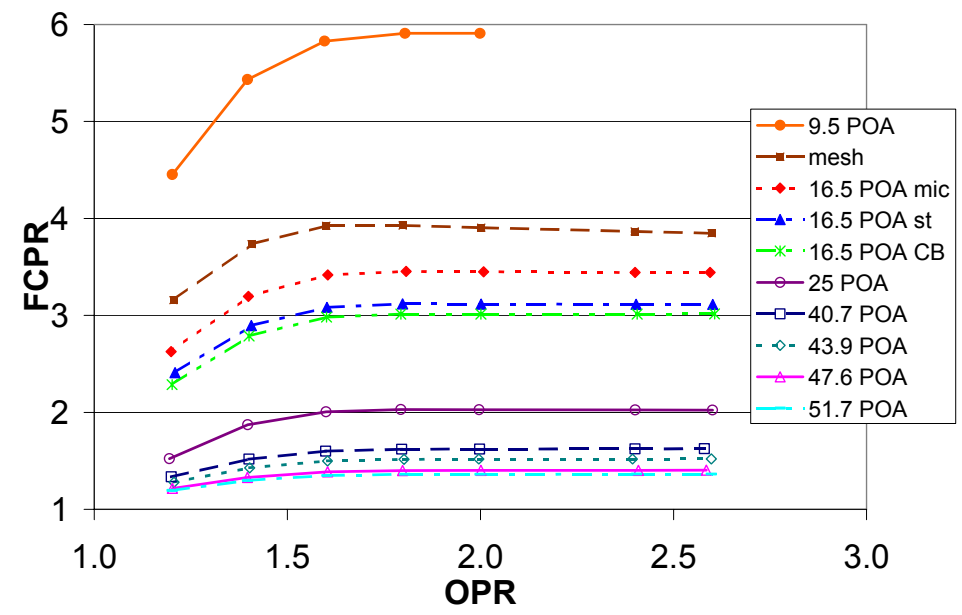

Figure 5. Flow conditioner pressure ratio versus operating pressure ratio for all flow conditioning devices at $\mathrm{AR}=0.43$.

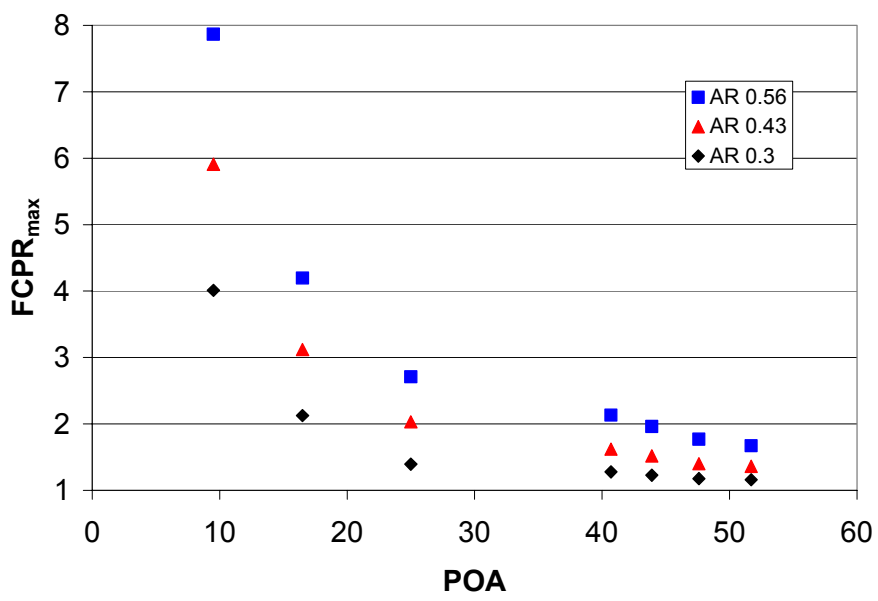

Figure 6. Maximum flow conditioner pressure ratio as a function of flow conditioner porosity.

8

American Institute of Aeronautics and Astronautics 


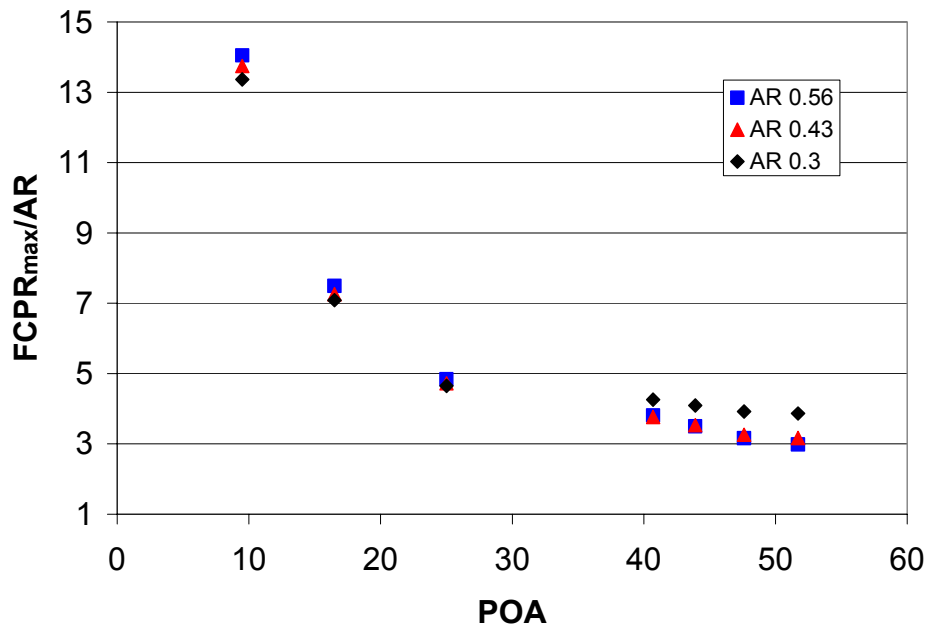

Figure 7. Maximum flow conditioner pressure ratio normalized by duct closure ratio as a function of flow conditioner porosity.

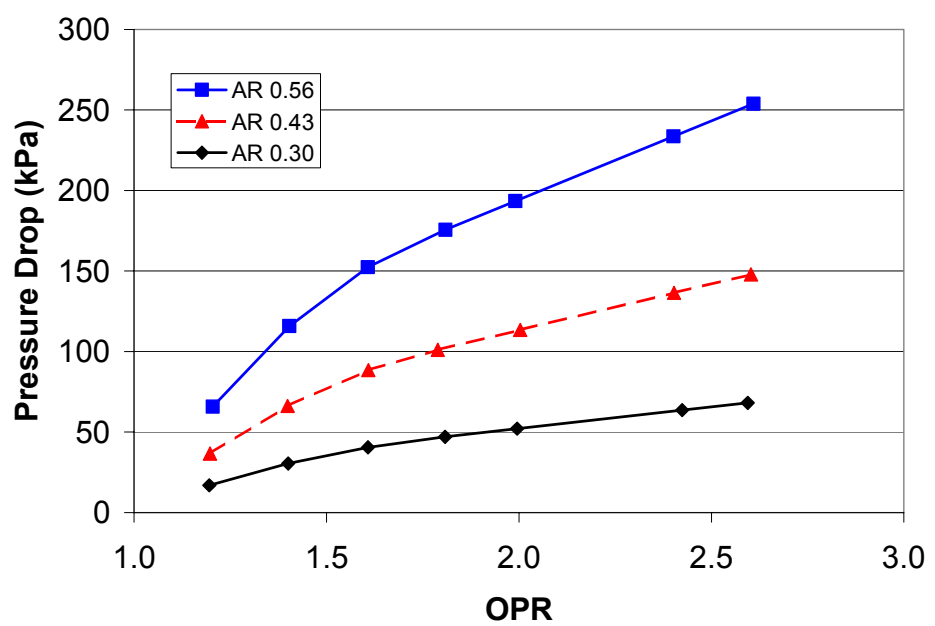

Figure 8. Stagnation pressure drop as a function of operating pressure ratio for 40.7 POA flow conditioner.

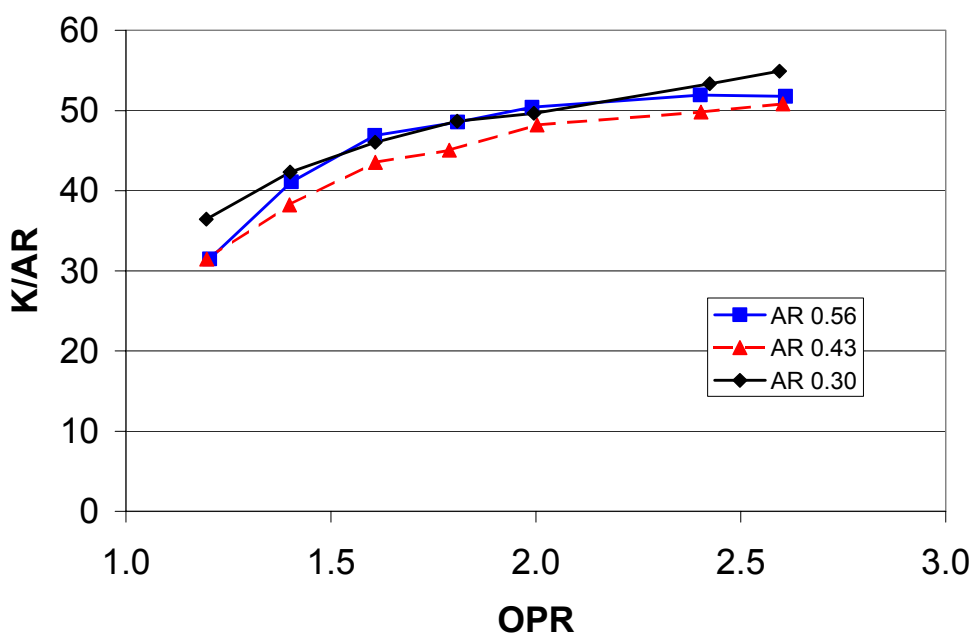

Figure 9. Pressure coefficient normalized by duct closure ratio as a function of operating pressure ratio for $\mathbf{4 0 . 7}$ POA flow conditioner.

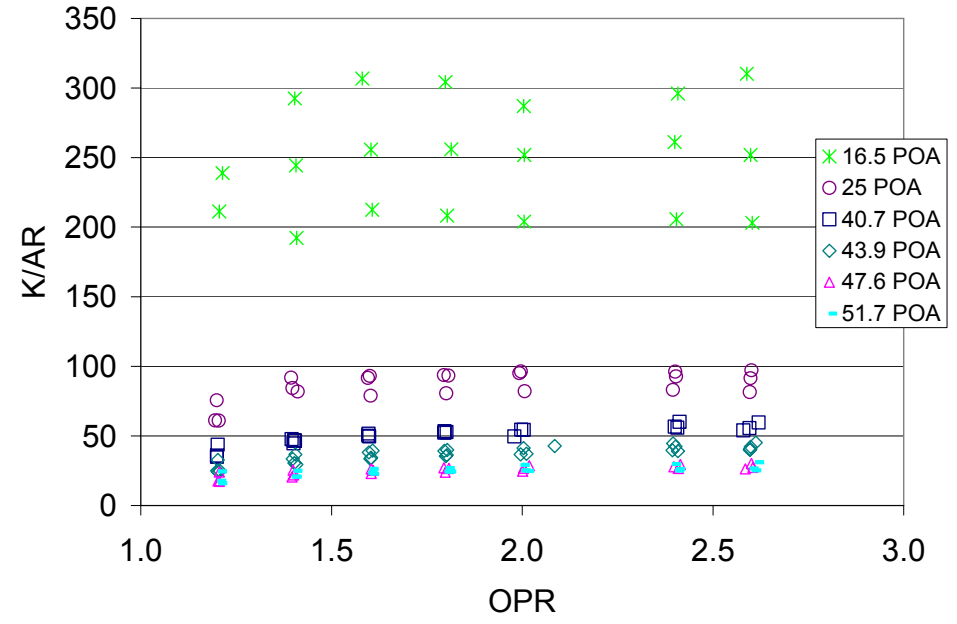

Figure 10. Normalized pressure coefficient for select flow conditioners as a function of operating pressure ratio.

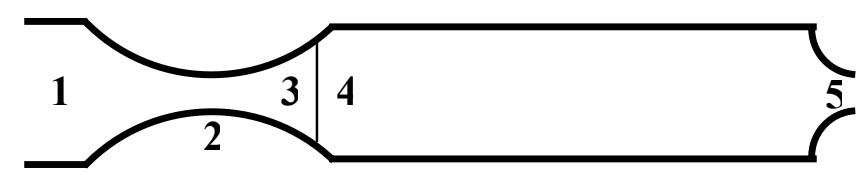

Figure 11. Compressible flow model of pressure losses for a choked flow conditioner.

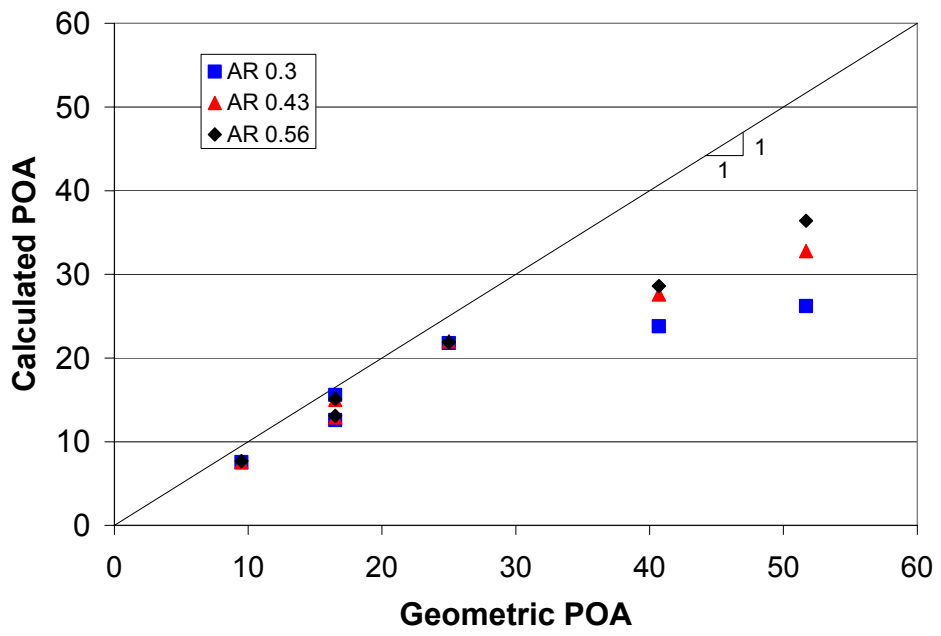

Figure 12. Calculated porosity as a function of geometric porosity using the compressible flow model. 


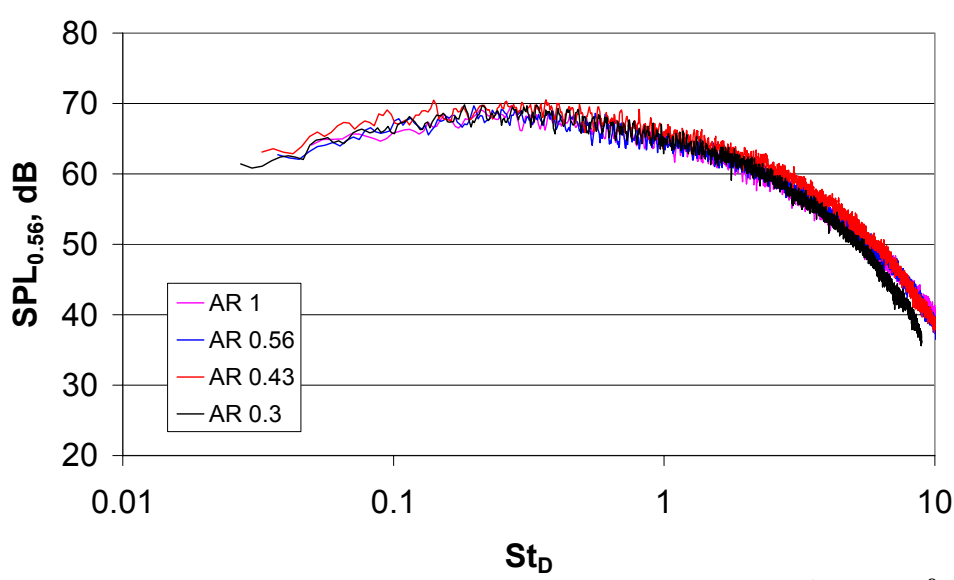

Figure 13. Far-field acoustic spectra at $\theta=90^{\circ}$ normalized to the amplitude of the $A R=0.56$ nozzle.

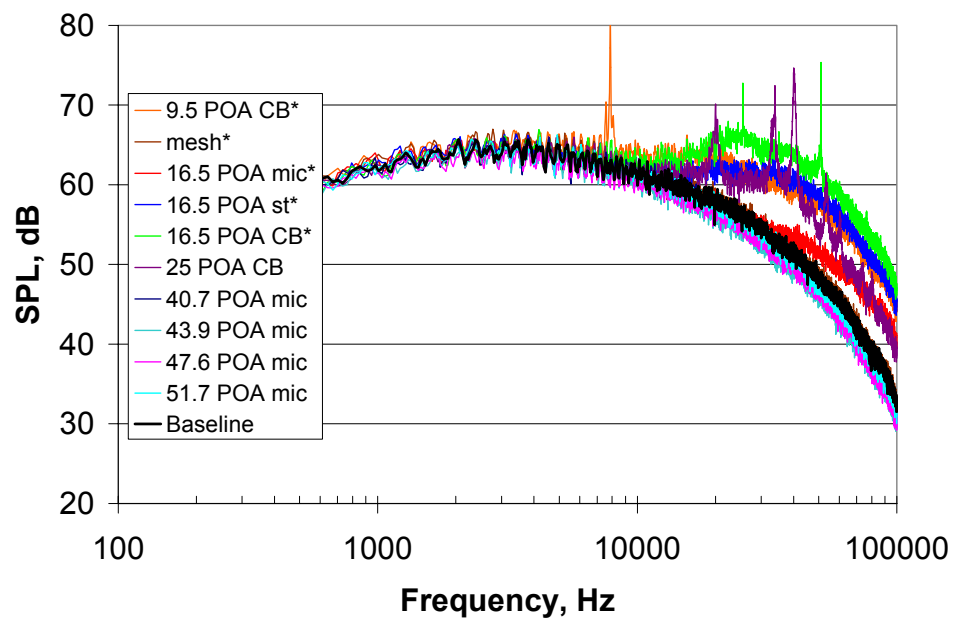

Figure 14. Far-field acoustic spectra at $\theta=90^{\circ}$ for all flow conditioners with $A R=0.3$ and $O P R=1.8$.

* indicates choked flow conditioner

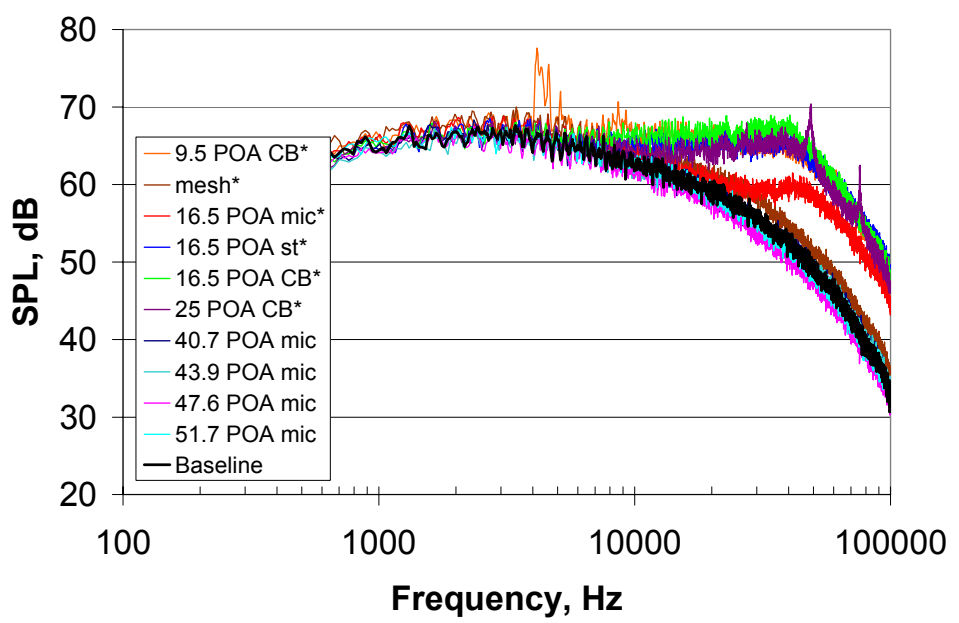

Figure 15. Far-field acoustic spectra at $\theta=90^{\circ}$ for all flow conditioners with $A R=0.43$ and $O P R=1.8$.

* indicates choked flow conditioner

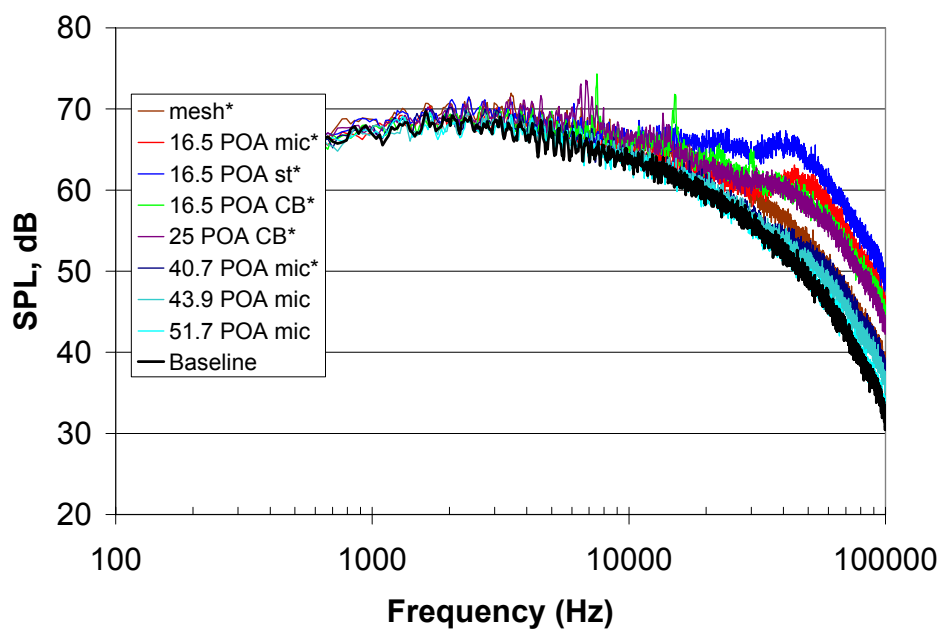

Figure 16. Far-field acoustic spectra at $\theta=90^{\circ}$ for all flow conditioners with $A R=0.56$ and $O P R=1.8$.

* indicates choked flow conditioner

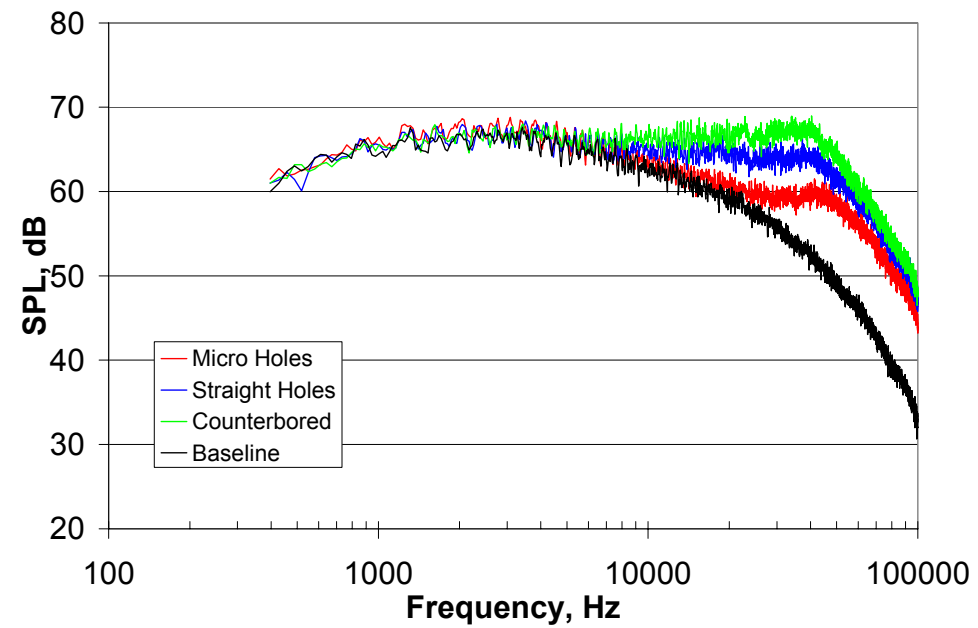

Figure 17. Far-field acoustic spectra at $\theta=90^{\circ}$ for 16.5 POA flow conditioners with $A R=0.43$ and $O P R=1.8$.

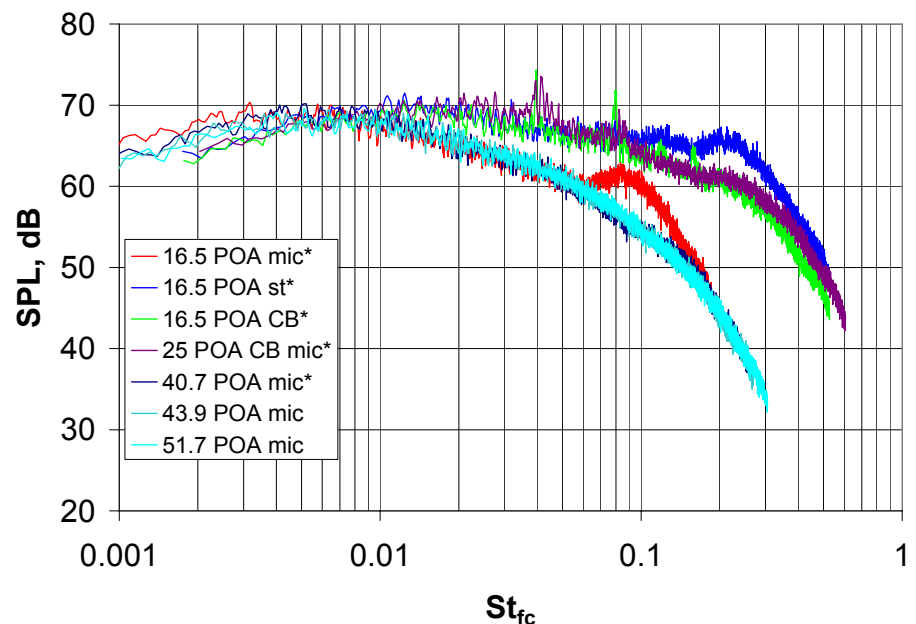

Figure 18. Far-field acoustic spectra at $\theta=90^{\circ}$ for all flow conditioners with $A R=0.56$ and $O P R=1.8$ as a function of Strouhal number based on flow conditioner exit velocity and hole size.

* indicates choked flow conditioner 